ARTICLE

Received 19 Feb 2016 | Accepted 10 May 2016 | Published 20 Jun 2016

DOI: $10.1038 /$ ncomms11895

OPEN

\title{
The power of a critical heat engine
}

\author{
Michele Campisi ${ }^{1} \&$ Rosario Fazio ${ }^{1,2}$
}

Since its inception about two centuries ago thermodynamics has sparkled continuous interest and fundamental questions. According to the second law no heat engine can have an efficiency larger than Carnot's efficiency. The latter can be achieved by the Carnot engine, which however ideally operates in infinite time, hence delivers null power. A currently open question is whether the Carnot efficiency can be achieved at finite power. Most of the previous works addressed this question within the Onsager matrix formalism of linear response theory. Here we pursue a different route based on finite-size-scaling theory. We focus on quantum Otto engines and show that when the working substance is at the verge of a second order phase transition diverging energy fluctuations can enable approaching the Carnot point without sacrificing power. The rate of such approach is dictated by the critical indices, thus showing the universal character of our analysis.

\footnotetext{
${ }^{1}$ NEST, Scuola Normale Superiore \& Istituto Nanoscienze-CNR, Pisa I-56126, Italy. ${ }^{2}$ ICTP, Strada Costiera 11, Trieste 34151, Italy. Correspondence and requests for materials should be addressed to M.C. (email: michele.campisi@sns.it).
} 
ncreasing the power output of a heat engine has a corresponding cost in terms of reduced efficiency $\eta$, or, in equivalent terms, a larger deviation $\Delta \eta=\eta^{\mathrm{C}}-\eta$ from the Carnot efficiency $\eta^{\mathrm{C}}$. A question that is currently the object of vigorous research efforts is whether it is possible to devise a heat engine that outputs finite power at Carnot efficiency ${ }^{1-5}$. In the following we address this question by focussing on the quantity

$$
\dot{\Pi} \doteq \frac{\mathcal{P}}{\Delta \eta}
$$

that is, the ratio of power output $\mathcal{P}$ over $\Delta \eta$. We shall call $\dot{\Pi}$ the performance rate. It is trivially possible to increase the power without affecting the efficiency by scaling the size of the working substance (WS): An array of $N$ identical engines working in parallel provides an $\mathrm{N}$-fold larger power than each of them, at the same efficiency. In this case the output work per cycle and efficiency scale as $\mathcal{P} \sim N$, and $\Delta \eta \sim 1$, consequently $\dot{\Pi} \sim N$ (here the symbol $\sim$ means 'scales as'). Note that this linear increase in performance rate does not represent any real gain as it is achieved at the cost of a corresponding linear increase of resources. The question is therefore 'Can the scaling of the performance rate $\dot{\Pi}$ be improved beyond linear' in order to have a true gain? Note that a positive answer implies (in an asymptotic sense) a positive answer to the fundamental question posed above: Imagine having a WS made up of $N$ constituents (or resources); if $\Pi \sim N^{1+a}$, with $a>0$, then one could approach Carnot efficiency as $\Delta \eta \sim \mathcal{P} / \dot{\Pi} \sim N^{-a} \rightarrow 0$ by increasing $N$, while keeping the 'power per resource' fixed, that is, $\mathcal{P} \sim N$.

In the following we make a substantial step towards answering the question above. The main idea that we pursue is that an interaction between the $N$ constituents of the WS could provide the extra scaling power that is needed for a positive resolution, see Fig. 1. In order to address that question quantitatively we consider a special engine cycle that is well studied in the literature (see, for example, refs 6-8 and references therein), namely, a quantum version of the Otto engine ${ }^{9-14}$, see Fig. 2. We show that a universal behaviour, with anomalous scaling of the performance rate

$$
\dot{\Pi} \sim N^{1+(\alpha-z v) /(d v)}
$$

emerges when the WS is on the verge of a second-order phase transition. Here $\alpha, v, z$ are the specific heat, correlation length and dynamical critical exponent, and $d$ is the number of dimensions of the WS. Note that the performance rate contains two concurrent contributions, one stemming from the scaling of the heat capacity, that is, the exponent $\alpha$, and the other stemming from the scaling of the relaxation time, that is, the exponent $z$. Since $d, v>0$, in order to have a more than linear behaviour of $\dot{\Pi}$

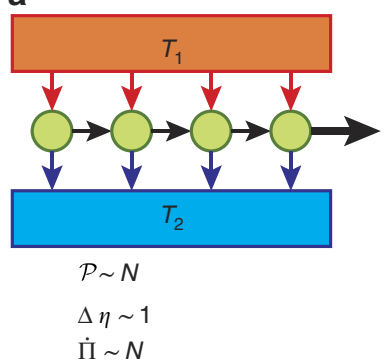

\section{b}

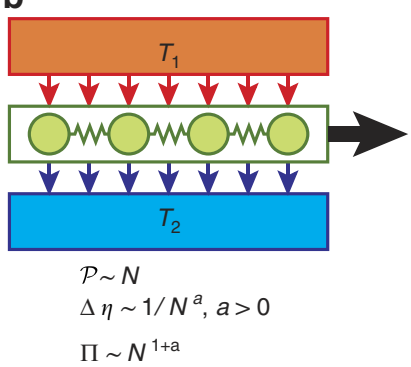

Figure 1 | Main idea at the basis of the results. (a) $N$ identical devices operating in parallel provide a power $\mathcal{P}$ that scales like $N$, at fixed efficiency. (b) When an interaction among the $N$ parallel devices is turned on, the approach to Carnot efficiency is enabled. one needs a WS with a critical point characterized by the inequality $\alpha-z v>0$. In fact, as we explain below in detail, the stronger condition

$$
\alpha-z v \geq 1
$$

would ensure the asymptotic approach towards the Carnot point without giving up power per resource. Is that possible? The finiteness of internal energy implies the bound $\alpha \leq 1$. Critical slowing down $(z \geq 0)$ would then imply $\alpha-z v \leq 1$ (for example, in the three-dimensional Ising model, $\alpha \simeq 0.12, v \simeq 0.63$ (ref. 15) and $z \simeq 2.35$ (ref. 16); hence $\alpha-z v \simeq-0.28$ ). A number of theoretical and experimental works report on the possibility of the exotic phenomenon of critical speeding up, $z \leq 0$ (refs 17-20). In this case the two terms might add up above unity (for example, recent experimental studies report $\alpha \simeq 0.38$ (ref. 21) and $z v \simeq-0.7$ (ref. 19); hence $\alpha-z v \simeq 1.08>1$, for $\left.\mathrm{Dy}_{2} \mathrm{Ti}_{2} \mathrm{O}_{7}\right)$. We conclude that there currently appears to be no fundamental reason hindering the possibility of approaching Carnot efficiency without giving up power per resource. This is certainly possible up to some threshold size $\bar{N}$ for which the weaker condition $\alpha-z v>0$ suffices. Below we illustrate how such powerful critical engines should be designed. At the heart of our result is the recognition that scaling of the performance, that is,

$$
\Pi=\frac{W_{\text {out }}}{\Delta \eta}
$$

where $W_{\text {out }}$ is the work output per cycle, is dictated by the heat capacity of the WS (which can notably diverge at the critical point), a crucial and simple fact that was never noticed before, see equation (9).

\section{Results}

N-body quantum Otto engine. The quantum Otto engine (see Fig. 2) is a four-stroke engine based on a WS with Hamiltonian

$$
H^{\mathrm{WS}}(t)=\lambda(t) K
$$

(Following the current literature we call these engines 'quantum Otto engines' although they bear no other quantum feature
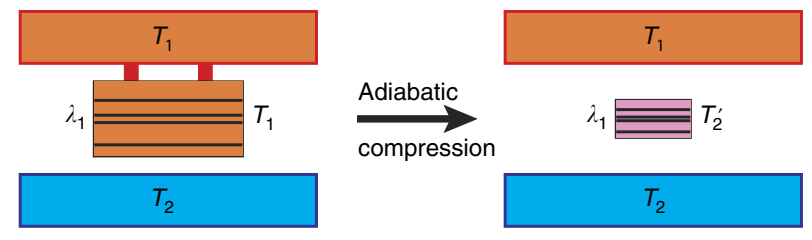

Thermalisation
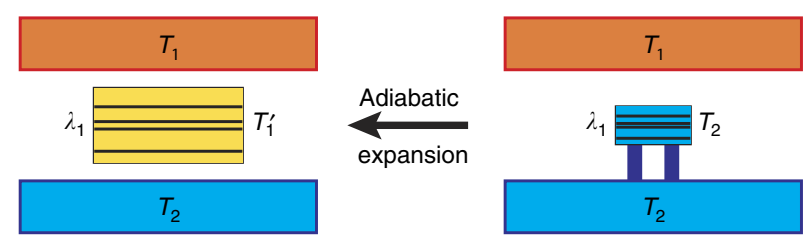

Figure 2 | A quantum Otto engine. At time $t=0$ the WS is in thermal equilibrium with bath 1 and $\lambda=\lambda_{1}$. During the first stroke the WS undergoes a thermally isolated transformation where the Hamiltonian switches from $H_{1}^{\mathrm{WS}}=\lambda_{1} \mathrm{~K}$ to $H_{2}^{\mathrm{WS}}=\lambda_{2} \mathrm{~K}$. The second stroke consists in thermalization with bath 2 , with $\lambda$ being kept fixed at $\lambda_{2}$. During the third stroke the Hamiltonian goes back to $H_{1}^{\text {wS }}=\lambda_{1} K$ while in thermal isolation. The fourth stroke consists in letting the system thermalize with bath 1 , with $\lambda$ being kept fixed at $\lambda_{1}$. 
besides the discreteness of the spectrum. Accordingly, there is nothing genuinely quantum in our treatment.) The heats released in the baths during the thermalization strokes are $Q_{i}=\mp \lambda_{i}\left[U_{K}\left(\beta_{2} \lambda_{2}\right)-U_{K}\left(\beta_{1} \lambda_{1}\right)\right]$, where $U_{K}(\theta)=$ $\operatorname{Tr} K e^{-\theta K} / \operatorname{Tr} e^{-\theta K}$ is the internal energy associated to the base Hamiltonian $K$ at inverse temperature $\theta$, and,-+ is for $i=1,2$, respectively. The work performed by the engine $W_{\text {out }}=Q_{1}+Q_{2}$ is thus

$$
W_{\text {out }}=\left(\lambda_{2}-\lambda_{1}\right)\left[U_{K}\left(\beta_{2} \lambda_{2}\right)-U_{K}\left(\beta_{1} \lambda_{1}\right)\right] .
$$

For $\beta_{1}<\beta_{2}$, the condition $W_{\text {out }} \geq 0, Q_{1} \geq 0$ defines the regime of operation of the engine as a heat engine, implying $\lambda_{2} / \lambda_{1} \geq \beta_{1} / \beta_{2}$. The efficiency is

$$
\eta=\frac{W_{\text {out }}}{Q_{1}}=1-\frac{\lambda_{2}}{\lambda_{1}} \leq 1-\frac{\beta_{1}}{\beta_{1}}=\eta^{\mathrm{C}} .
$$

The efficiency is smaller than $\eta^{\mathrm{C}}$, in accordance with the heat engine fluctuation relation ${ }^{22,23}$.

Using equation (7) the output work of the quantum Otto engine can be expressed as a function of $\lambda_{1}, \beta_{1}, \beta_{2}$ and $\Delta \eta=\lambda_{2} / \lambda_{1}-\beta_{1} / \beta_{2}$ as

$$
W_{\text {out }}=\lambda_{1}\left(\Delta \eta-\eta^{\mathrm{C}}\right)\left[U_{K}\left(\beta_{1} \lambda_{1}+\beta_{2} \lambda_{1} \Delta \eta\right)-U_{K}\left(\beta_{1} \lambda_{1}\right)\right] .
$$

This expression allows us to study the performance $\Pi$. In the region where linear approximation holds, that is, $\Delta \eta \ll 1$ (namely $\beta_{1} \lambda_{1}$ is close to $\beta_{2} \lambda_{2}$ ), the scaling of the engine's performance $\Pi$ with $N$ is given by the scaling of $\partial W_{\text {out }} /\left.\partial \Delta \eta\right|_{\Delta \eta=0}$, that is, the slope of $W_{\text {out }}(\Delta \eta)$ at the origin. This is illustrated in Fig. 3. In the case of $N$ devices in parallel it is $W_{\text {out }}=N W_{\text {out }}^{\text {single }}$ (here $W_{\text {out }}^{\text {single }}$ denotes the work of each single device); hence trivially $\partial W_{\text {out }} /\left.\partial \Delta \eta\right|_{\Delta \eta=0} \sim \Pi \sim N$, and, accordingly $\dot{\Pi} \sim N$, as we noted before. It follows that in order to boost the scaling of the performance it is necessary to have the slope $\partial W_{\text {out }} /\left.\partial \Delta \eta\right|_{\Delta \eta=0}$ to scale more than linearly.

By taking the derivative of equation (8) with respect to $\Delta \eta$ we obtain the central relation

$$
\left.\Pi \sim \frac{\partial W_{\text {out }}}{\partial \Delta \eta}\right|_{\Delta \eta=0} \sim N c_{K}\left(\beta_{1} \lambda_{1}\right)
$$

where $c_{K}(\theta)=-(1 / N) \theta^{2} \partial U_{K} / \partial \theta$ is the specific heat of the WS. To understand the physics behind the emergence of equation (9),

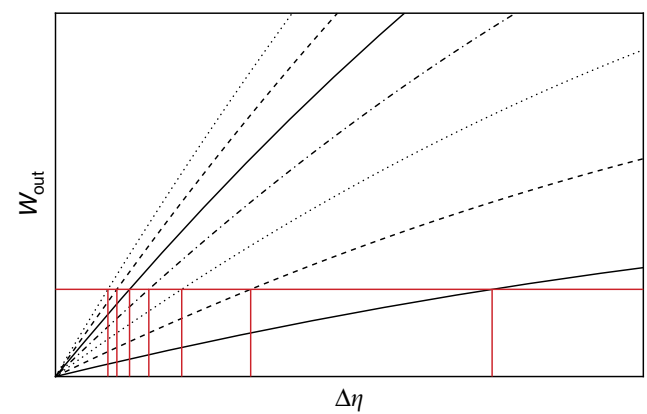

Figure 3 | Graphical demonstration of equation (9). The graph shows various plots of $W_{\text {out }}(\Delta \eta)$ for increasing values of $N$ (from lower to upper curves). At a fixed work output (horizontal red line), the curves are intercepted at decreasing values of $\Delta \eta$. If the slope at the origin increases with a power $N^{a}, \partial W_{\text {out }} /\left.\partial \Delta \eta\right|_{\Delta \eta=0} \sim N^{a}$; then, provided for larger and larger $N$ the intercept occurs in the region where the linear approximation holds for the $N$ th curve, the approach towards $\Delta \eta=0$ occurs as $1 / N^{a}$; hence $\Pi=W_{\text {out }} / \Delta \eta \sim N^{a} \sim \partial W_{\text {out }} /\left.\partial \Delta \eta\right|_{\Delta \eta=0}$. Similarly, one might increase (lower) the value of $W_{\text {out }}$ with $N$, for example, as $W_{\text {out }}(\Delta \eta)=w N^{b}$. Accordingly, provided for larger and larger $N$ the intercept still occurs in the region where the linear approximation holds, the approach towards $\Delta \eta=0$ occurs as $1 / N^{a-b}$. Still, $\Pi=W_{\text {out }} / \Delta \eta \sim N^{a} \sim \partial W_{\text {out }} /\left.\partial \Delta \eta\right|_{\Delta \eta=0}$. consider working at some point that is very close to the Carnot point, that is, chose $\lambda_{1}$ and $\lambda_{2}$ so that their ratio $\lambda_{1} / \lambda_{2}$ is very close to $\beta_{2} / \beta_{1}$. After the adiabatic compression stroke the WS reaches a new temperature $T_{2}^{\prime}=1 /\left(k_{B} \beta_{2}^{\prime}\right)$, which is very close to the cold bath temperature $T_{2}=1 /\left(k_{B} \beta_{2}\right)$ : $T_{2}^{\prime}=T_{1} \lambda_{2} / \lambda_{1}=T_{2}+\Delta T_{2}$, with a small $\Delta T_{2}$. The larger the heat capacity $C_{K}=N c_{K}$ of the WS, at the thermalization point, the larger the heat exchanged $Q_{2}=C_{K} \Delta T_{2}$ during the subsequent thermalization with the cold bath. Likewise for the subsequent expansion and thermalization. Since $W_{\text {out }}=Q_{1}+Q_{2}$, the larger the heat capacity the larger the work output, and accordingly the larger the performance.

Equation (9) tells us that in order to achieve super-linear scaling of the performance, one needs a WS with an anomalous scaling of the specific heat. Recall that for ordinary substances the heat capacity is extensive, $C_{K} \sim N$, namely $c_{K}=C_{K} / N \sim 1$. What is needed for improved performance is $c_{K} \sim N^{a}$, with some $a>0$. This can happen at the verge of a phase transition. The physical reason is that at a phase transition finite exchanges of heat are accompanied by infinitesimal changes of temperature, that is, the specific heat diverges. This is because at the transition point the energy intake is not employed to heat up but rather to make the change of phase.

For a second-order phase transition, finite size scaling predicts a peak in the specific heat whose height and width scale, respectively, as $\bar{c}_{K} \sim N^{\alpha /(d v)}$ and $\delta \sim N^{-1 /(d v)}$ (ref. 24) where $d$ is the dimensionality of the system, and $\alpha$ and $v$ are the specific heat and the correlation length critical exponents. Accordingly we predict the possibility of a boosted scaling of the perfmormance $\left.\Pi \sim N^{1+\alpha}\right)(d v)$. Writing the performance rate as $\dot{\Pi}=\mathcal{P} / \Delta \eta=$ $W_{\text {out }} /(\mathcal{T} \Delta \eta)=\Pi / \mathcal{T}$ we see that its scaling is determined by the scaling of $\Pi$ and of the cycle time $\mathcal{T}$. The latter is dominated by the thermalization time ${ }^{2}$, which, according to finite-size scaling theory scales with the dynamical critical exponent $z$ as $\mathcal{T}_{\text {relax }} \sim N^{z / d}$ (ref. 25). This gives equation (2).

Critical engine design. We illustrate how a quantum Otto engine can be designed to achieve the predicted performance rate in equation (2). Let the substance be described by a Hamiltonian $K$, displaying, in the infinite size limit, a second-order phase transition at the inverse critical temperature $\theta_{\mathrm{C}}$. Let the two baths have the inverse temperatures $\beta_{1}$ and $\beta_{2}$. We assume we can stretch/compress the spectrum of the WS and implement accordingly the time-dependent Hamiltonian in equation (5) with $\lambda(t) \in\left[\lambda_{1}, \lambda_{2}\right]$. When $\lambda$ takes on the value $\lambda_{i}$, the critical temperature is rescaled to $\beta_{\mathrm{C}}^{i}=\theta_{\mathrm{C}} / \lambda_{i}$. We choose, for example, $\lambda_{1}$ so that $\beta_{1}=\beta_{\mathrm{C}}^{1}$ (that is $\lambda_{1}=\theta_{\mathrm{C}} / \beta_{\mathrm{C}}^{1}$ ). In this way the temperature of bath 1 coincides with the critical temperature of $H_{1}^{\mathrm{WS}}=\lambda_{1} K$. We next choose $\lambda_{2}$ as the solution of $W_{\text {out }}=N^{1+z / d_{w}}$, for some fixed $w$. That gives the corresponding efficiency $\eta=1-\lambda_{2} / \lambda_{1}$. Note that since $\mathcal{T} \sim N^{z / d}$, the power per constituent is fixed: $\mathcal{P}=W_{\text {out }} / \mathcal{T} \sim N$. Since the slope of the graph $W(\Delta \eta)$ grows with the heat capacity as $\Pi \sim N^{1+\alpha /(d v)}$, we have $\Delta \eta=W_{\text {out }} / \Pi \sim N^{1+z / d} / N^{1+\alpha /(d v)} \sim N^{-(\alpha-v z) / d v}$. Hence if $\alpha-v z>0$ the solution $\lambda_{2}$ gets closer and closer to $\lambda_{1} \beta_{1} / \beta_{2}$. Accordingly the efficiency $\eta=1-\lambda_{2} / \lambda_{1}$ gets closer and closer to $\eta^{\mathrm{C}}=1-\beta_{1} / \beta_{2}$. As discussed in the caption of Fig. 3 , the previous statement is correct as long as $\lambda_{2}$ falls in the region of validity of the linear approximation (that is, in graphical terms, if the corresponding $\Delta \eta$ falls in the region where the curve $W(\Delta \eta)$ is well approximated by a straight line passing through the origin). The extension of that region corresponds to the width of the specific heat peak, which, as mentioned above, universally scales as $\delta \sim N^{-1 /(d v)}$. So, in order to keep pace with the shrinking of the linear approximation region, the approach towards $\Delta \eta=0$ should 
a

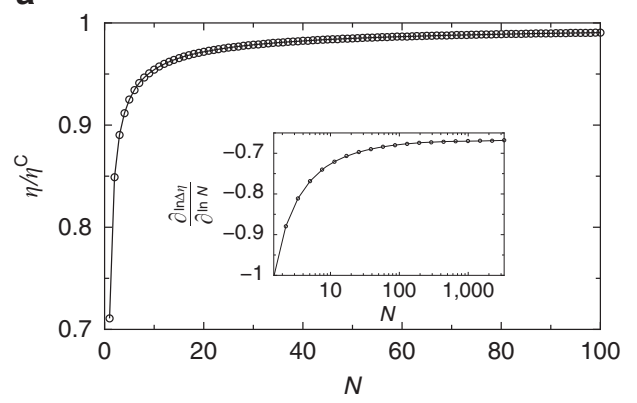

b

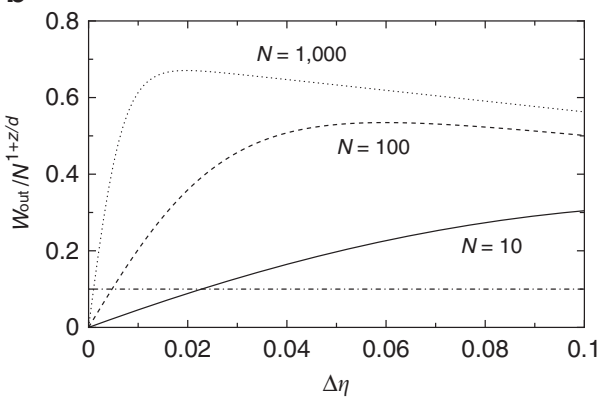

Figure 4 | Efficiency and shrinking of linear region in a critical sped-up engine. (a) Approach towards the Carnot point of a critical working substance characterized by equation (3) at a fixed power per constituent. Plots were calculated with critical indices reported recently for $\mathrm{Dy}_{2} \mathrm{Ti}_{2} \mathrm{O}_{7}$, namely $\alpha \simeq 0.38$ (ref. 21) and $z v \simeq-0.7$ (ref. 19). The value of $v$ was obtained from the relation $v=(2-\alpha) / d$ (ref. 27). Carnot point was at $\eta^{C}=1 / 2$ and $w=W_{\text {out }} / N^{1+z / d}=0.1$. The inset shows the quantity $\partial \ln \Delta \eta / \partial \ln N$ as a function of $N$, that is, the exponent characterizing the approach to the Carnot point that reaches the expected value $-(\alpha-z v) /(d v) \simeq-0.67$. (b) Rescaled output work $W_{\text {out }} / N^{1+z / d}$ as a function of $\Delta \eta$. Note that the linear region shrinks around the origin of axes as $N$ increases, and that the intercept with $W_{\text {out }} / N^{1+z / d}=0.1$ occurs within that region for all curves.

be not slower than $N^{-1 /(d v)}$. Since that would occur at a pace of $N^{-(\alpha-v z) /(d v)}$, the condition ensuring that it actually asymptotically occurs is given by equation (3).

In Fig. 4a we illustrate how in such a case the engine design that we have described above actually results in an asymptotic approach towards the Carnot point, at a fixed power per constituent. Figure $4 \mathrm{~b}$ illustrates the shrinking of the linear region, due to the narrowing of the peak width. In making Fig. 4 we have taken full advantage of one of the most striking aspects of our analysis, namely its universality. The details of the specific model are not essential, all that counts are the critical exponents. The specific heat peak reads $c_{K}(\theta)=N^{\alpha /(d v)} \theta^{2} U_{1} \Delta U f\left(\Delta U\left(\theta-\theta_{\mathrm{C}}\right) N^{1 /(d v)}\right)$, where $f(x)$ is some bell-shaped function (its exact shape is not relevant). The corresponding internal energy of the WS around the critical point reads $U_{K}(\theta)=U_{0}-N^{1+(\alpha-1) /(d v)} U_{1} F\left(\Delta U\left(\theta-\theta_{\mathrm{C}}\right) N^{1 /(d v)}\right)$, where $F^{\prime}(x)=f(x)$. The plots are obtained by using the latter formula, with equation (6) used to evaluate the work output, with the choice $f(x)=\operatorname{sech}^{2}(x), \quad F(x)=\tanh (x)$ and $U_{1}=\Delta U_{1}=1$. The plot illustrates the approach towards Carnot efficiency at a fixed power per constituent, with the predicted scaling exponent $-(\alpha-z v) /(d v)$.

\section{Discussion}

The idea that phase transitions could enable the attainment of Carnot efficiency at finite power was previously hinted by Polettini et al. ${ }^{5}$, but was never pursued before. The present work confirms that intuition in a fully fledged way based on universality and finite-size scaling theory and most importantly by accounting for the first time for the effect of criticality on the time of operation hence of the power.

As is clear from Fig. 3, independent of how the slope $\partial W_{\text {out }} /\left.\partial \Delta \eta\right|_{\Delta \eta=0}$ scales, $W_{\text {out }}(\Delta \eta=0)=0$; hence, exactly at the Carnot point all quantum Otto engines deliver null work and hence null power. Our statement should be accordingly understood in a weaker asymptotic sense, namely, that it is possible to get as close as one wants to the Carnot point without giving up the power per constituent. This is the viewpoint that also inspires ref. 2 .

We remark that our linear condition $\Delta \eta \ll 1$ substantially differs from the condition of linear response regime (that is, $\beta_{2}-\beta_{1} \ll \beta_{2}$ or $\left.\eta \ll 1\right)$ that was investigated previously in refs $1,3-5$. In our case the two temperatures need not be close to each other.

We stress that all obstacles hindering the realization of the critical powerful Carnot engines appear to be of technological nature, rather than fundamental. One major difficulty stems from the necessity of implementing the Hamiltonian (5) containing a global coupling $\lambda(t)$, which can be very challenging in practice. Another difficulty stems from the fact that in order to be able to asymptotically approach the Carnot point, one should accordingly have an increasing degree of accuracy with which $\lambda_{1}$ and $\lambda_{2}$ are controlled.

Lastly, it is worth stressing that the result in equation (9) holds in general as a consequence of the linear approximation valid for $\Delta \eta \ll 1$, and is accordingly not restricted to the case of critical phenomena. This tells that one route towards the improvement of the performance of a WS is to increase its specific heat. That could be achieved by increasing its number of constituents, a possibility that we have investigated here, or by manipulating any other parameter entering the Hamiltonian $K$. Recently reported cases of improved performances ${ }^{26}$ can in fact be interpreted in terms of increased heat capacity.

Data availability. The authors declare that all data supporting the findings of this study are available within the article.

\section{References}

1. Benenti, G., Saito, K. \& Casati, G. 'Thermodynamic bounds on efficiency for systems with broken time-reversal symmetry'. Phys. Rev. Lett. 106, 230602 (2011).

2. Allahverdyan, A. E., Hovhannisyan, K. V., Melkikh, A. V. \& Gevorkian, S. G 'Carnot cycle at finite power: attainability of maximal efficiency'. Phys. Rev. Lett. 111, 050601 (2013).

3. Proesmans, K. \& Van den Broeck, C. 'On-sager coefficients in periodically driven systems'. Phys. Rev. Lett. 115, 090601 (2015).

4. Brandner, K., Saito, K. \& Seifert, U. 'Thermodynamics of micro- and nano-systems driven by periodic temperature variations'. Phys. Rev. X 5, 031019 (2015).

5. Polettini, M., Verley, G. \& Esposito, M. 'Efficiency statistics at all times: Carnot limit at finite power'. Phys. Rev. Lett. 114, 050601 (2015).

6. Benenti, G., Casati, G., Prosen, T. \& Saito, K. Fundamental aspects of steady state heat to work conversion. Preprint at arXiv:1311.4430 (2013).

7. Kosloff, R. \& Levy, A. 'Quantum heat engines and refrigerators: continuous devices'. Annu. Rev. Phys. Chem. 65, 365-393 (2014).

8. Gelbwaser-Klimovsky, D., Niedenzu, W. \& Kurizki, G. 'Chapter TwelveThermodynamics of Quantum Systems under Dynamical Control' 329-407 (Academic Press, 2015).

9. Scully, M. O. 'Quantum afterburner: Improving the efficiency of an ideal heat engine'. Phys. Rev. Lett. 88, 050602 (2002).

10. Feldmann, T. \& Kosloff, R. 'Quantum four-stroke heat engine: Thermodynamic observables in a model with intrinsic friction'. Phys. Rev. E 68, 016101 (2003).

11. Quan, H., Liu, Y.-X., Sun, C. \& Nori, F. 'Quantum thermodynamic cycles and quantum heat engines'. Phys. Rev. E 76, 031105 (2007).

12. Niskanen, A. O., Nakamura, Y. \& Pekola, J. P. 'Information entropic superconducting microcooler'. Phys. Rev. B 76, 174523 (2007). 
13. Allahverdyan, A. E., Johal, R. S. \& Mahler, G. 'Work extremum principle: Structure and function of quantum heat engines'. Phys. Rev. E 77, 041118 (2008).

14. Rofinagel, J., Abah, O., Schmidt-Kaler, F., Singer, K. \& Lutz, E. 'Nanoscale heat engine beyond the carnot limit'. Phys. Rev. Lett. 112, 030602 (2014).

15. Pelissetto, A. \& Vicari, E. 'Critical phenomena and renormalization-group theory'. Phys. Rep 368, 549-727 (2002).

16. Matz, R., Hunter, D. L. \& Jan, N. 'The dynamic critical exponent of the three-dimensional Ising model'. J. Stat. Phys. 74, 903-908 (1994).

17. Zappoli, B. et al. 'Anomalous heat transport by the piston effect in supercritical fluids under zero gravity'. Phys. Rev. A 41, 2264-2267 (1990).

18. Boukari, H., Briggs, M. E., Shaumeyer, J. N. \& Gammon, R. W. 'Critical speeding up observed'. Phys. Rev. Lett. 65, 2654-2657 (1990).

19. Grams, C. P., Valldor, M., Garst, M. \& Hemberger, J. 'Critical speeding-up in the magne-toelectric response of spin-ice near its monopole liquid-gas transition'. Nat. Commun. 5, 4853 (2014).

20. Tavora, M., Rosch, A. \& Mitra, A. 'Quench dynamics of one-dimensional interacting bosons in a disordered potential: elastic dephasing and critical speeding-up of thermalization'. Phys. Rev. Lett. 113, 010601 (2014).

21. Higashinaka, R., Fukazawa, H., Deguchi, K. \& Maeno, Y. 'Low temperature specific heat of dy2ti2o7 in the kagome ice state'. J. Phys. Soc. Jpn. 73, 2845-2850 (2004)

22. Campisi, M. 'Fluctuation relation for quantum heat engines and refrigerators'. J. Phys. A: Math. Theor. 47, 245001 (2014).

23. Campisi, M., Pekola, J. \& Fazio, R. 'Nonequilibrium fluctuations in quantum heat engines: theory, example, and possible solid state experiments'. New J. Phys. 17, 035012 (2015).

24. Fisher, M. E. \& Barber, M. N. 'Scaling theory for finite-size effects in the critical region'. Phys. Rev. Lett. 28, 1516-1519 (1972).

25. Suzuki, M. 'Static and dynamic finite-size scaling theory based on the renormalization group approach’. Prog. Theor. Phys. 58, 1142-1150 (1977).
26. Cakmak, S., Altintas, F. \& Mustecaplioglu, O. E. Lipkin-Meshkov-Glick Model in a quantum Otto Cycle. Preprint at arXiv:1510.04495 (2015).

27. Huang, K. Statistical Mechanics 2nd edn (Wiley, 1987).

\section{Acknowledgements}

This research was supported by UNICREDIT, the 7th European Community Framework Programme under grant agreements no. 623085 (IEF-NeQuFlux), no. 600645 (IP-SIQS), no. 618074 (STREP-TERMIQ) and by the COST action MP1209 'Thermodynamics in the quantum regime'.

\section{Author contributions}

M.C. conceived the idea. M.C. and R.F. conducted the work, analysed the results and drew the conclusions.

\section{Additional information}

Competing financial interests: The authors declare no competing financial interests

Reprints and permission information is available online at http://npg.nature.com/ reprintsandpermissions/

How to cite this article: Campisi, M. and Fazio, R. The power of a critical heat engine. Nat. Commun. 7:11895 doi: 10.1038/ncomms11895 (2016).

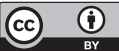

This work is licensed under a Creative Commons Attribution 4.0 International License. The images or other third party material in this article are included in the article's Creative Commons license, unless indicated otherwise in the credit line; if the material is not included under the Creative Commons license, users will need to obtain permission from the license holder to reproduce the material. To view a copy of this license, visit http://creativecommons.org/licenses/by/4.0/ 